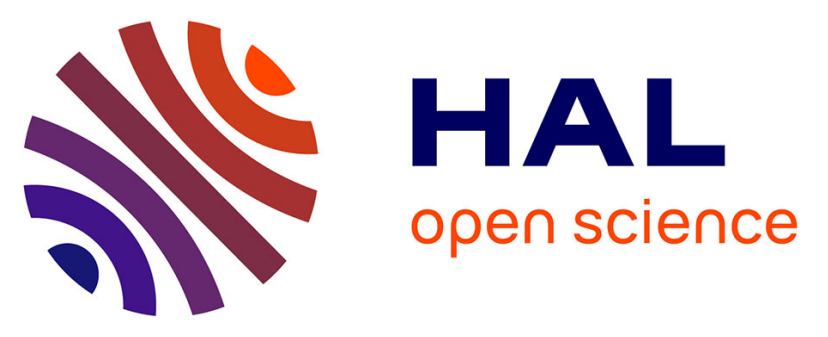

\title{
A comparative study of degradation mechanisms of PBSA and PHBV under laboratoryscale composting conditions
}

Salomez Mélanie, Matthieu George, Pascale Fabre, Touchaleaume François, Cesar Guy, Lajarrige Anaïs, Emmanuelle Gastaldi

\section{To cite this version:}

Salomez Mélanie, Matthieu George, Pascale Fabre, Touchaleaume François, Cesar Guy, et al.. A comparative study of degradation mechanisms of PBSA and PHBV under laboratoryscale composting conditions. Polymer Degradation and Stability, 2019, 167, pp.102-113. 10.1016/j.polymdegradstab.2019.06.025 . hal-03070279

\author{
HAL Id: hal-03070279 \\ https://hal.science/hal-03070279
}

Submitted on 15 Dec 2020

HAL is a multi-disciplinary open access archive for the deposit and dissemination of scientific research documents, whether they are published or not. The documents may come from teaching and research institutions in France or abroad, or from public or private research centers.
L'archive ouverte pluridisciplinaire HAL, est destinée au dépôt et à la diffusion de documents scientifiques de niveau recherche, publiés ou non, émanant des établissements d'enseignement et de recherche français ou étrangers, des laboratoires publics ou privés.

\section{(ㅇ)(1) $\$$}

Distributed under a Creative Commons Attribution - NonCommercial - NoDerivatives 44.0 
1 A comparative study of degradation mechanisms of PBSA and PHBV under laboratory-

\title{
scale composting conditions
}

\author{
SALOMEZ Mélanie ${ }^{1}$, GEORGE Matthieu $^{3}$, FABRE Pascale ${ }^{3}$, TOUCHALEAUME \\ François $^{1}$, CESAR Guy ${ }^{2}$, LAJARRIGE Anaïs ${ }^{1}$, GASTALDI Emmanuelle $^{1 *}$ \\ ${ }^{1}$ UMR IATE Ingénierie des Agropolymères et Technologies Emergentes, Campus INRA-SupAgro, 2 place Pierre \\ Viala, 34060 Montpellier, France \\ ${ }^{2}$ SERPBIO, Université Bretagne Sud, 56321 Lorient, France \\ ${ }^{3}$ Laboratoire Charles Coulomb, CNRS UMR-5221, Université de Montpellier, Place E. Bataillon, 34095 \\ Montpellier, France
}

\section{ABSTRACT}

Biodegradable plastics appear as one promising means to help solving the increasing issue of environmental pollution by plastics. The present study aims at comparing the biodegradation mechanisms of two promising biodegradable plastics, PHBV Poly(3hydroxybutyrate-co-3-hydroxyvalerate) and PBSA Poly(butylene succinate-co-adipate) with the objective to provide a better understanding of the mechanisms involved and identify the most relevant indicators to follow biodegradation. For this purpose, the progress of the biodegradation process was monitored under controlled composting conditions at the laboratory scale at $58^{\circ} \mathrm{C}$ using several methodological approaches for evaluating polymer degradation. Indicators of the extent of material disappearance based on respirometry and mass loss were combined to other indicators evidencing the morphological, structural and chemical modifications induced at the surface or in the bulk of the material as surface erosion by MEB and AFM, decrease of molecular weight by GPC, crystallinity changes by DSC and chemical changes by ATR-FTIR. As expected, both polymers were rapidly biodegraded in less than 80 days. However, in spite of its higher molecular weight and degree of crystallinity PHBV degraded faster than PBSA, which led to suggest that different biodegradation mechanisms would be involved. At this regard, a two-phase scenario was proposed for each polymer on the strength of all the degradation-induced changes observed the polymer surface and in its bulk. Based on these two scenarios, the discrepancy in biodegradation rate between PHBV and PBSA would be essentially attributed to significant differences in crystals morphology and spatial organization of both polymers.

Regarding the relevance of the different indicators studied, mass loss stood out as the most relevant and accurate indicator to assess the disappearance of material especially when combined with respirometry and mineralization kinetics assessment. Besides, indicators focusing on the surface changes as SEM, AFM and POM were emphasized since seen as powerful tools to evidence morphological changes at different scales. At last, changes in thermal properties as crystallinity rate and melting temperature, even if complex to interpret due to the wide range of interdependent mechanisms they bring into play 
appeared as inescapable tools for improving the understanding of the underlying mechanisms involved in polymer biodegradation.

KEYWORDS: biodegradation, crystallinity, erosion, compost, Poly(3-hydroxybutyrate-co-3hydroxyvalerate), Poly(butylene succinate-co-adipate)

\section{INTRODUCTION}

With the increase of the global population, the demand for plastic materials in every aspect of life and industry has become tremendous. As a result, the most of plastics produced worldwide since 1950 (around 79\%) has been accumulated in landfills or in the environment. To face this environmental concern, which invariably causes injuries to ecosystems equilibriums, biodegradable plastics, whether they derive from renewable feedstocks or petroleum, are seen as promising means to help solving plastic waste management issues. However, the global production capacities of biodegradable thermoplastics remains still very low, estimated at $0.91 \mathrm{MT}$ in 2018 (Source: European Bioplastics 2018) compared to the 348MT of total plastics produced worldwide in 2017 (Source: Plastics Europe Market Research Group (PEMRG)). The biggest industrial sector using plastics is the packaging sector (44.8\% of total polymer resin production), they are also the primary waste producer generating half of plastic wastes [1]. The most used polymers for packaging are polyethylene (PE) and polypropylene (PP). But, their chemical stability is excessive in comparison with the material lifespan required in the most of common everyday life usages where plastic is needed (food packaging, single use products, cosmetics, etc.).

Among the biodegradable plastics suitable for replacing conventional plastics i.e. prone to satisfy the requirements of a plastic for daily use in terms of functional and environmental properties, aliphatic polyesters like polyhydroxyalkanoates (PHAs) and poly(butylene succinate-co-adipate) (PBSA) are both interesting candidates. Nowadays global production of PHAs and PBS/PBSA is respectively of $3.2 \%$ and $7.4 \%$ of all biodegradable polymers behind starch blends, PLA and PBAT (Source: European bioplastic 2018). These two families have the advantage to be totally of partially biosourced, which ensure a lower carbon footprint compared to petrochemically derived plastics. PHAs belong to the family of biopolymers synthesized by several bacteria as intracellular carbon and energy storage granules. A wide variety of prokaryotic organisms can accumulate PHAs from 30 to $80 \%$ of their cellular dry weight by fermentation using various renewable feedstocks. PHAs have 150 different types of monomers allowing the tuning of their physical properties from crystalline-brittle to softsticky materials depending on the length of the side aliphatic chain at the chiral carbon [2]. PHAs and more specifically Poly(3-hydroxybutyrate-co-3-hydroxyvalerate) known as PHBV have interesting oxygen and water vapour barrier properties compared to other biodegradable polymers, thus making it a good candidate for food packaging applications [3] [4]. PBS and its copolymers are a family of biodegradable polymers with excellent 
biodegradability, thermoplastic processability and balanced mechanical properties [5]. Its relatively good processability via a broad range of conventional techniques (i.e. sheet extrusion, injection molding, thermoforming, blow molding, foaming, fiber spinning, and filament) renders it suitable for the production of various materials designed for daily use (packaging, automotive, textile, and sports and leisure industries). Poly(butylene succinateco-adipate) known as PBSA is synthesized from 1,4-butanediol, succinic acid and adipic acid monomers by copolymerization. In the past, all the monomers constituting PBSA were exclusively derived from fossil raw materials. However, with the development of biorefinery throughout the world, these key biobased chemical building blocks are more and more obtained by fermentative production routes based on renewable feedstocks of second generation (dextrose, glucose, sucrose, biobased glycerol, and vegetable oil).

PHBV and PBSA polyesters have very good biodegradability performances in various environments compared to other biodegradable polymers [6-8]. Poly(3-hydroxybutyrate) is actually considered as a suitable alternative for cellulose as a reference material in the standards of biodegradation for soil and water environments, respectively NF EN 17033 and ISO DIS 14852. Copolymerization is one of the strategies used to improve biodegradability and tune functional performances. Increasing hydroxyvalerate content in PHBV decreases its melting temperature, elastic modulus and tensile strength with higher elongation at break up to $970 \%$ [9] and improved biodegradability [10]. In the same way, the copolymerisation of PBS with butylene adipate monomers up to $60 \%$ increases chain mobility and biodegradability of the copolymer by lowering both crystallinity and melting temperature [11]. Within available commercial PHBV, P(3HB-co-3HV) material at molar percentage of HV is very brittle with high elastic modulus, low tensile strength and an elongation at break around 3\%, thus making it a strong and hard material [12]. Among commercially available PBSA polyesters, P(BS-Co-BA) at $20 \% \mathrm{~mol}$ BA displays a good compromise between biodegradation and mechanical performances (tensile strength and elongation at break of 430\%) [5].

The ability of polymers to biodegrade is driven by several parameters related to intrinsic chemical and physical properties as emphasized by enzymatic degradation studies [13]. Among them, not only their chemical composition and first order structure (molecular weight and distribution, degree of branching) are expected to be involved but also their physical state including higher order structures (glass transition temperature, melting temperature, modulus of elasticity, degree of crystallinity, crystals size and structure) together with their surface properties (hydrophobicity, roughness, specific surface). Beside the role played by the intrinsic material properties on biodegradation performances, the environmental conditions including both abiotic phenomena (UV, oxidation, mechanical stress) and biotic ones are also important parameters to be focused on. While abiotic phenomena lead to the damage and fragmentation of a polymer by oxidation and hydrolysis mechanisms only biotic phenomena will result into the complete mineralization of a 
polymer. The primary prerequisite that drives the biodegradation of plastics lies on the adherence of microorganisms on the surface of plastics followed by the colonization of the exposed surface and the formation of a biofilm. Afterwards, plastics are enzymatically degraded in a two-step process: first the enzyme binds to the surface of the plastic substrate and secondly, the enzymatic catalysis of the hydrolytic cleavage resulting in the reduction of the polymer chain length into low molecular weight oligomers, dimers and monomers [14]. Once polymer chains are short enough, they can be assimilated by microorganisms and ultimately converted aerobically into biomass, water and $\mathrm{CO}_{2}$. The second requirement that should be fulfilled for considering a polymer as biodegradable in a given environment is thus the presence in the medium of microorganisms able to synthesize and release (exo)enzymes able to degrade this polymer. In that respect, as established for a long time by Tokiwa and Suzuki (1977), the ability of polyesters to biodegrade comes from their susceptibility to be hydrolyzed by lipases or esterases, which are ubiquitous enzymes in the environment.

To our knowledge, no studies have compared the biodegradation performance of different families of biodegradable polymers in controlled environmental conditions while also studying the underlying mechanisms. The results of Yang et al. [8] have shown that PBSA and PCL biodegraded better than other polymers (PP, PLLA, PBS) in the same compost but the performance and mechanisms of biodegradation were not further investigated. The study of Mercier [7] compared the degradation of different polymers (EVOH, PP, PBAT, PET, PBS, sclPHA, PLLA, PA66) in an uncontrolled compost simulating home composting conditions (average temperature of $13^{\circ} \mathrm{C}$ ) and found that PBS and scl-PHA were the polymers which degraded the most with a mass loss of 5.5 and $8 \%$, respectively after 450 days of incubation. Discrepancy in degradation performances of the polymers tested was mainly attributed to the formation of higher cell density biofilms and their specific surface properties. Other studies have focused on the degradation of a single polymer in laboratory composting conditions. However, results are difficult to compare due to the use of different experimental conditions, i.e. different kind of compost and inoculum, and also because of different processes to obtain polymer films (hot pressing, casting or extrusion). Biodegradation of PHBV has been extensively studied in composting conditions [15-20] in contrast to studies dealing with the biodegradation of PBSA, which are still scarce $[8,21]$. Furthermore, it is rare to see different methodological approaches to evaluate polymer biodegradation. Most of these studies generally focused on changes in mass loss without monitoring in parallel the carbon dioxide released by the material during its degradation. It is however worth noting that evidencing polymer mass loss does not guaranty its final assimilation by microorganisms.

In this context, the present study aims to compare the biodegradation performance and mechanisms of films made of PHBV and PBSA under controlled composting conditions at the laboratory scale. This comparison between these two promising biodegradable polymers could help to identify key limiting factors driving biodegradation. For this purpose, initial 
surface properties of both films were characterized and the progress of the biodegradation process was monitored using several methodological approaches for evaluating polymer degradation. Indicators of the extent of material disappearance (assessed by respirometry and mass loss measurement) were combined to other indicators evidencing the morphological, structural and chemical modifications induced at the surface or in the bulk of the material (surface erosion, chain molecular weight decrease, crystallinity and chemical changes). Correlating such approaches could not only help identifying the key parameters explaining the differences in biodegradability between the two polymers but would also contribute to evidence indicators that could be considered as relevant quantitative descriptors for evaluating the progress of the biodegradation process.

\section{MATERIALS AND METHODS}

\subsection{Materials}

The PHBV and PBSA pellets were purchased from Natureplast (France) under the trade names PHIO02 and PBE001, respectively. PHBV contains 1-3 mol\% of hydroxyvalerate (HV) and contains boron nitride as nucleating agent. PBSA contains $21+/-1$ mol\% of butylene adipate (BA) and is $31 \%$ biosourced. Cellulose microcrystalline powder, soda and hydrochloric acid were purchased from Merck. Formamide and diiodomethane were provided by Acros organics (Geel, Belgium), ethylene glycol and hexamethyldisilazane were purchased from Aldrich chemical Co. Inc. (Milwaukee, USA).

PHBV and PBSA pellets were extruded using a twin-screw extruder (Thermo ScientificTM EuroLab 16) with an L/D ratio of 40 and a screw diameter of $16 \mathrm{~mm}$ equipped with a flat die and calendering. The processing conditions used were a screw speed of $200 \mathrm{rpm}$, a flow rate of $1 \mathrm{~kg} / \mathrm{h}$ and temperature profiles from 140 to $180^{\circ} \mathrm{C}$ for PHBV and 100 to $135^{\circ} \mathrm{C}$ for PBSA. PHBV and PBSA films displayed a thickness of $175+/-25 \mu \mathrm{m}$ and $210+/-25 \mu \mathrm{m}$, respectively.

\subsection{Biodegradation tests}

Respirometric tests were conducted using a method adapted from NF ISO 14855 standard to evaluate the biodegradation kinetic of PHBV and PBSA films in composting conditions. For this purpose, mature green compost was collected in the waste management centre of Aspiran (France) and sieved through $5 \mathrm{~mm}$ meshes. Biodegradation tests were carried out in cylindrical hermetic glass vessels $(1000 \mathrm{~mL}$ capacity) containing three small open polypropylene flasks ( $60 \mathrm{~mL}$ capacity). The first flask contained $6 \mathrm{~g}$ of wet compost with final water content of $50 \%$, (pH of 8.05 and $\mathrm{C} / \mathrm{N}$ ratio of 26.6 ), mixed with an equivalent of $50 \mathrm{mg}$ carbon of each tested film previously cut in $8 \times 8 \mathrm{~mm}$ pieces. The second flask contained 30 $\mathrm{mL} \mathrm{NaOH}$ solution $(0.1 \mathrm{M})$ to trap the $\mathrm{CO}_{2}$ produced by microorganisms, and the third flask contained distilled water in order to maintain the relative humidity at $100 \%$ inside the vessel. The blank control was composed only of compost. Cellulose microcrystalline powder was used as positive control. The blank and positive controls were done in triplicate whereas each test sample of PHBV and PBSA film was repeated twelve times. All the samples were incubated in the dark at $58 \pm 1^{\circ} \mathrm{C}$ during 120 days. At selected time interval, $\mathrm{NaOH}$ flasks were 
removed from the vessel to be titrated and were then replaced by new flasks containing $\mathrm{NaOH}$. At the same time and during the first 41 days of incubation, one flask containing a test sample of both films was recovered to ensure mass loss measurements and further analysis. The compost humidity was maintained constant by adding distilled water all along the experiment. The percentage of mineralization of a test sample was determined as the ratio of the amount of carbon dioxide released from the material mineralization related to the maximum theoretical amount of carbon dioxide that could be released by the test material using Equation (1):

Mineralization $(\%)=\frac{n_{\mathrm{CO} 2 \text { test }}-n_{\mathrm{CO} 2 \text { blank }}}{n_{\mathrm{CO} 2 \text { theoretical }}}$

Where, $\mathrm{n}_{\mathrm{CO} 2 \text { test }}$ is the amount of $\mathrm{CO}_{2}$ released by the respiration of the compost medium and the mineralization of the material, $\mathrm{n}_{\mathrm{CO} 2}$ blank is the amount of $\mathrm{CO}_{2}$ released by the compost medium only, and $\mathrm{n}_{\mathrm{CO} 2}$ theoretical, the maximal theoretical amount of $\mathrm{CO}_{2}$ released by the mineralization of the material calculated by using its carbon content. Biodegradation curves with no priming effect were selected and modelled using Hill and Boltzmann equations [22].

\subsection{Mass loss}

The PHBV and PBSA films removed from the compost throughout the biodegradation process (at day $6,10,15,20,29,41$ ) were carefully soaked 5 min in ethanol $70 \%$ and then rinsed with distilled water to remove all the particles. Finally, samples were dried overnight at $60^{\circ} \mathrm{C}$ under vacuum and weighted using an electro balance to determine the percentage of mass loss using Equation (2):

Mass loss $(\%)=\frac{m_{0}-m_{f}}{m_{0}} \times 100$

Where, $m_{0}$ is the mass of the material before biodegradation and $m_{f}$ the mass after biodegradation.

Prior to the subsequent analyses (see below), an ultra-sound treatment was applied to the dried specimens to remove any residual organic matter and microorganisms from the surface. For this purpose a sonicator (Qsonica Q700) with a microtip probe was used during 1 min repeated 3 times (amplitude of 30\%) and a rest time of 2 min between each run.

\subsection{Surface properties: contact angle measurements and atomic force microscopy}

Surface properties of pristine PHBV and PBSA films were determined by contact angle measurements performed at $25^{\circ} \mathrm{C}$ using a goniometer (Digidrop, GBX, France) equipped with a CCD camera ( 25 frames/sec) and the GBX software (Windrop, GBX, France). The dispersive $\left(\gamma_{s}{ }^{d}\right)$ and polar $\left(\gamma_{s}{ }^{A B}\right)$ components of the solid surface tension were evaluated by applying the Owens-Wendt approach [26]. For this purpose, four reference liquids were used: formamide, diiodomethane and ethylene glycol. Their respective surface tension components were taken from the literature and are listed in Table 1 [23-24]. 
Atomic force microscopy (AFM) was performed on each sample to get accurate insight of the initial surface state of both polymers. Characteristic image of respective area, $10 \times 10 \mu \mathrm{m}^{2}$ and $50 \times 50 \mu \mathrm{m}^{2}$, were acquired for each sample using a Nanoscope $V$ (Bruker instruments, Madisson, WI, United States) in contact mode (Binnig et al., 1986) and standard silicon probes (Bruker, DNP). Root mean square (RMS) roughness of the polymer surface was measured for each scale with Gwyddion software after subtraction of the average plane.

\subsection{Scanning Electron Microscopy (SEM)}

SEM observations were performed using a field emission scanning electron microscope (FESEM S-4500, Hitachi, Japan) with an acceleration voltage of $2 \mathrm{kV}$ secondary electrons. PHBV and PBSA film samples were either directly mounted on stub using carbon conductive tape and then coated with gold/palladium by ion sputtering, or previously cryo-fractured under liquid nitrogen, depending on the observation that was done on the film surface or its cross-section, respectively.

\subsection{Fourier Transform InfraRed spectroscopy (FTIR)}

PHBV and PBSA films were analysed using an infrared spectrometer (Thermo Scientific, Nicolet 6700) and a DTGS-KBr detector in Attenuated Total Reflectance (ATR) mode. FTIRATR spectra ranged from 4000 to $800 \mathrm{~cm}^{-1}$ with a resolution of $2 \mathrm{~cm}^{-1}$ and were averaged over 32 scans. Carbonyl index were calculated by normalizing the area of each peak over the area of a reference peak, respectively $1473 \mathrm{~cm}^{-1}$ for PBSA (-CH- symmetric deformation [25]) and $1379 \mathrm{~cm}^{-1}$ for PHBV (-CH3 symmetric wagging $\left.[26,27]\right)$ after correction of the spectral baseline.

\subsection{Molecular weight}

The PHBV and PBSA film samples prepared as described above were dissolved in chloroform at a concentration of $5 \mathrm{mg} / \mathrm{ml}$. PBSA dissolved immediately whereas PHBV samples needed to be heated to $50^{\circ} \mathrm{C}$ and agitated for $1-2 \mathrm{~h}$ until complete dissolution arrived. Resulting solutions were then filtered through a $0.45 \mu \mathrm{m}$ polytetrafluoroethylene (PTFE) syringe filter. Molecular weights were measured by GPC (Waters system) at $35{ }^{\circ} \mathrm{C}$ using a PLgel Mixed C$5 \mu \mathrm{m}-2 \times 300 \mathrm{~m}$ column and a refractive index (RI) detector. Chloroform was used as an eluent at a flow rate of $1 \mathrm{ml} / \mathrm{min}$. The number-average $(\mathrm{Mn})$ and weight-average (Mw) molecular weights were calculated using a calibration curve from polystyrene standards. The scission index [28] was calculated from the following Equation (3):

$S I=\frac{M n_{(t=0)}}{M n_{(t)}}-1$

Where $\mathrm{Mn}_{(\mathrm{t}=0)}$ and $\mathrm{Mn}_{(\mathrm{t})}$ are the initial number-average molecular weight and the numberaverage molecular weight at a given time of biodegradation, respectively. In our study, the scission index was calculated between day 6 and day 41 .

\subsection{Differential Scanning Calorimetry (DSC)}


Thermal analyses were carried out on 5-8 mg of PBSA and PHBV films collected from the compost at different time intervals using a TA instrument DSC Q200 under nitrogen atmosphere. A thermal ramp of $5^{\circ} \mathrm{C} / \mathrm{min}$ was used during the first run of heating and the second run of cooling with a temperature ranging from $-40^{\circ} \mathrm{C}$ to $150^{\circ} \mathrm{C}$ and $-40^{\circ} \mathrm{C}$ to $200^{\circ} \mathrm{C}$, respectively. The crystallinity degree of samples was calculated from thermograms using Equation (4):

$X_{c}(\%)=\frac{\Delta H_{m}}{\Delta H_{m}^{0}} \times 100$

Where, $\Delta H_{m}$ is the melting enthalpy and $\Delta H_{m}^{0}$ the melting enthalpy of the polymer supposed to be $100 \%$ crystalline, i.e $110.3 \mathrm{~J} / \mathrm{g}$ for PBSA [29] and $146 \mathrm{~J} / \mathrm{g}$ for PHBV [30]. The highest peak of the melting scan of pristine PHBV and PBSA was used to calculate their respective melting temperature.

\section{RESULTS}

\section{1. (Bio)degradation kinetics under laboratory-scale composting conditions}

The biodegradation rate of PHBV and PBSA films was monitored by concomitantly measuring the released carbon dioxide (Fig. 1) and the weight loss (Fig. 2). To ensure an accurate comparison between the two measurements, several respirometric tests have been launched simultaneously, one of them being periodically interrupted for weight loss measurement and complementary analysis. Beyond 40 days of incubation, measurements on plastic specimens could not be further performed. The degradation was too far advanced to ensure the proper collection of the sample, the films breaking into small pieces, which became impossible to recover from the composting medium. In contrast to weight loss measurement, respirometric tests allowed monitoring biodegradation until the complete mineralisation of the material.

The biodegradation curves and weight loss measurements (Fig. 1-2) confirmed that both polymers were fully biodegradable, i.e. that the entire material was fully mineralised into carbon dioxide attesting its final assimilation by microorganisms. This was confirmed by the plateau phase reached by the evolution of carbon dioxide similar to the positive control (cellulose), which reflected that no further biodegradation was expected. The mineralization rates of PHBV and PBSA specimens reached $100 \%$ after $70-90$ days of incubation (Fig. 1).

The evolution of mass loss in Figure 1 could be divided in two phases, each being characterized by a different kinetic of degradation. During the first phase (0-20 days), the degradation of PHBV was faster than for PBSA and increased exponentially over time. At the end of this phase, mass loss was five times higher for PHBV than for PBSA, with 50\% against $10 \%$, respectively. After 20 days of incubation, both degradation rates changed with a slow down for PHBV whereas an acceleration was observed for PBSA. This change in degradation 
Table 1

Figure 1

Figure 2

kinetics could also be evidenced on the mineralisation curves, resulting in two different types of modelling; a (single) sigmoidal shape according to Hill equation for PHBV versus a double sigmoidal shape curve fitting with Boltzmann equation for PBSA. Despite the increased rate of degradation of PBSA, its overall degradation kinetic remained slightly slower than these of PHBV. As a consequence, the plateau phase of respirometric curves was reached earlier for PHBV than for PBSA. As an illustration of such differences no pieces of PHBV film could be recovered in the compost in contrast to $5-10 \%$ residual material weighted for PBSA at the last sampling. The good correlation found between respirometric and mass loss curves until 40 days indicated that the mass loss of both material was essentially due to the conversion of organic carbon in carbon dioxide without implying any leaching phenomenon.

\subsection{Surface morphological and macrostructural modifications}

Surface polarity, RMS roughness and 3D topography of both pristine polymer films have been investigated using contact angle measurements and AFM (Table 1). Such properties are known to be key parameters driving the adhesion of microorganisms and controlling biofilm formation on a material surface [31, 32]. The surface properties of pristine PHBV and PBSA films indicated that PBSA was slightly more hydrophilic than PHBV as reflected by a higher relative polar component $\left(\gamma_{\mathrm{S}}{ }^{\mathrm{AB}} / \gamma_{\mathrm{S}}=13 \%\right.$ for PBSA against $10 \%$ for PHBV) and also a higher water wettability as indicated by a contact angle value $(\theta)$ of $68.2^{\circ}$ for PBSA against $76^{\circ}$ for PHBV. However, despite these small but significant differences, it could be assumed that both polymers exhibited rather close surface properties in terms of surface energy.

By contrast, the surface morphology of the pristine PHBV and PBSA films evidenced by AFM exhibited contrasted scales of structuring with a different surface roughness depending on the scale considered (Table 1). As shown on AFM 3D pictures, PHBV surface was covered by compacted spherical structures displaying a diameter around 10-15 $\mu \mathrm{m}$ and that could be ascribed to spherulites [33]. Such a structural organization led the RMS value to decrease from 230 to $58 \mathrm{~nm}$ when reducing the scale from 50 to $10 \mu \mathrm{m}$. In contrast, PBSA presented a surface made of numerous asperities of a few micrometres height whatever the considered scale. Thus, even if PHBV and PBSA exhibited quite similar roughness values at $50 \mu \mathrm{m}$ scale, AFM images and roughness values obtained at $10 \mu \mathrm{m}$ scale clearly showed that PBSA had initially a surface 20-times rougher than PHBV. 
To follow macroscopic modifications occurring at the surface of both polymers during the progress of the biodegradation process, MEB images were performed on films sampled from the compost medium at different time intervals. MEB images in figure 3 showed the timeevolution of the surface erosion for PHBV and PBSA. Comparison between both polymers revealed a stronger degradation of the PHBV film surface than for PBSA. Such surface erosion that could be compared to an enzymatic etching evidenced significant differences in the crystal organisation of each polymer. In PHBV, spherulites with highly ordered lamellae became clearly visible only 10 days after starting the incubation in compost (Figure 3c). This primary stage of surface erosion could be ascribed to a faster degradation of amorphous phase in comparison with crystalline phase as generally reported in literature regarding enzymatic degradation of plastics [13]. By contrast, the surface erosion of PBSA revealed a different kind a structure. In early degradation stages, flat layers of crystals were observed (Figure 3f).

\section{Figure 3}

Images obtained at a lower magnification (Figure 4) indicated that this erosion phenomenon occurred heterogeneously at the surface leading to the formation of erosion patterns. Comparison between both polymers revealed footprints of microbial filaments on the surface of PHBV films as seen in figure 4a, whereas these filaments were absent or scarcely observed on PBSA films. These footprints appeared as the starting points of surface degradation as evidenced on figure $4 \mathrm{~b}$. Colonization of PHBV by fungi could thus have been more important than for PBSA. Such differences could be linked with the differences in roughness evidenced at small scale by AFM (Table 1 ). It has been previously reported that microbial communities of plastics, the plastisphere, would be specific for each plastics [7].

Figure 4

To complete this microstructural investigation, a macroscopic analysis of the degradationinduced physical changes was undertaken at a higher scale of observation. As illustrated in Figure 5, two different degradation behaviours were observed with the formation of holes for PHBV whereas PBSA fragmented after around 20 days of incubation. These differences in morphology were confirmed by SEM images of both films incubated 41 days in compost medium revealing that PHBV displayed a clear tendency to become porous with an eroded shape, whereas PBSA tended to fracture with the formation of sharp edges (Figure 6). This propensity to fragment as a distinctive feature to PBSA has already been reported by Puchalski et al. [21] when studying the degradation of PBSA in compost conditions.

Figure 5

Figure 6

\subsection{Surface physico-chemical modifications during composting}


ATR-FTIR measurements were performed at the surface of PBSA and PHBV films (from 0.5 to $5 \mu \mathrm{m}$ deep depending of the wavelength) collected from the compost medium over time during 40 days with the aim to evidence the chemical modifications induced by the biodegradation process. Based on the structure of polyesters, hydroxyl $(\mathrm{OH})$ and carbonyl $(C=O)$ groups could be used as tools to study degradation and hydrolysis of ester linkages $[16,19,34]$. In the present study, the peak usually ascribed to $\mathrm{OH}$ groups $\left(3350 \mathrm{~cm}^{-1}\right.$ free $-\mathrm{OH}$ stretching) was not taken into account since rather affiliated to the presence of water. The presence of this peak was also associated with a peak at $1650 \mathrm{~cm}^{-1}$ corresponding to the $C=O$ stretch of amide functions probably due to the presence of proteins from microorganisms (results not shown). To remedy these artefacts, all the films samples were subjected to an ultrasound treatment and a drying prior to FTIR analysis to remove microorganisms and water.

Evolution of carbonyl index of PHBV and PBSA films during incubation in compost medium is given in Figure 7. The carbonyl index of PHBV $\left(1770-1700 \mathrm{~cm}^{-1}\right)$ was stable during the first 20 days of degradation in compost suggesting no accumulation at the film surface of carbonyl groups resulting from the ester linkage hydrolysis (Fig. 7). Thus, it can be supposed that the oligomers produced by the enzymatic hydrolysis of the polymer were probably immediately assimilated by microorganisms or leached in the compost medium, hydrolysis and erosion being probably concomitant in this first phase for PHBV. Luo et al. [16] and Weng et al. [19] who studied biodegradation in compost medium of PHBV and PHAs, respectively, also concluded that the chemical structure of residual material surface remained unchanged during the degradation process. By contrast during the second degradation phase ( $>20$ days) the carbonyl index of PHBV started to decrease. This could suggest that after 20 days oligomers produced by the enzymatic hydrolysis of ester linkages remained at the film surface since not easy to release in the medium and/or not rapidly consumed by bacteria. The evolution of carbonyl index of PHBV during this second phase could be related with the slow down in mass loss and respirometry that also occurred after 20 days.

For PBSA, a different behaviour was observed at the film surface with a decrease of the carbonyl index (1770-1690 $\mathrm{cm}^{-1}$ ) during the first 20 days and no change afterwards (Fig. 7). Enzymatic hydrolysis of the ester linkages during this first phase did not lead to a leaching of the oligomers that would increase mass loss. This could explain the lower erosion rate of PBSA during the first phase. During the second phase ( $>20$ days), the decrease of the carbonyl index slowed down suggesting that the hydrolysis was concomitant with the leaching of oligomers chains. This could explain the higher rate of mass loss in this second phase of degradation.

Figure 7

\subsection{Bulk structural modifications}


Changes in bulk properties of both polymer films during biodegradation were firstly assessed through the evolution of their number-average molecular weight $(\mathrm{Mn})$ molecular weight as a function of degradation time in compost (Fig. 8). The results indicated PHBV was initially roughly twice higher than for PBSA with $\mathrm{Mn}$ values of 94000 versus $44000 \pm 3000 \mathrm{~g} / \mathrm{mol}$. A linear decrease in $\mathrm{Mn}$ values was observed according to time, which was accompanied a more or less pronounced reduction in the polydispersity depending on the considered polymer. The polydispersity index of PBSA was initially equal to 7.5 , and then it rapidly decreased to 3.3 at 6 days and finally stabilized around 2.6 after 41 days incubation in compost. Regarding PHBV, changes in dispersity were weaker with values varying from 2.38 to 2.2 during the degradation time in compost. The decrease in $\mathrm{Mn}$ is a typical consequence of the biodegradation process that reflected bulk changes rather than surface erosion that would be induced by degrading enzymes [35] [36] [21]. This assertion is based on the rather low specific surface of the polymer films tested in the present study (thickness of 175-210 $\mu \mathrm{m})$ together with the fact that enzymes produced in the compost medium were not supposed to diffuse into the bulk of the material due to their steric hindrance. As a consequence the decrease in Mn mainly provided an indication of the involvement of hydrolytic chain scission mechanisms resulting in a noticeable size chains reduction.

However, the decrease in polydispersity index led to suggest that another degradation mechanism would be implied. According to Puchalski [21] the reduction of polydispersity index would be due to the enzymatic etching of oligomers from the film surface, these latters would be small enough to diffuse and would be finally released in the compost medium. The combination of a relatively slow hydrolysis of high $\mathrm{Mn}$ polymers and a faster enzymatic cleavage of oligomers and low Mn polymers would lead $\mathrm{Mn}$ and polydispersity to decrease concomitantly. This also accounted for the rapid decrease of the polydispersity index of PBSA during the first days of incubation in compost. The distribution profile of polymer chains exhibited a population of short chains $(<4000 \mathrm{~g} / \mathrm{mol})$ that quickly disappeared during incubation in compost. These short chains were probably expected to diffuse through the material and were quickly leached, which is in accordance with previous studies [37] [38].

Figure 8

During degradation in compost medium the scission index also increased linearly with a rate 2.5 times higher for PBSA than for PBHV (Fig. 9). Scission index value being closely associated with the ability of ester linkages to be hydrolysed by the water contained in the compost medium, this indicator is thus expected to increase with the water permeability and water diffusion of polymers. According to data reported in literature PBSA exhibited a higher water permeability and water diffusion coefficient than PHBV, with $P$ values of 6787 barrer against 149 barrer [39] [40], and $D_{0}$ values of $2.010^{-8} \mathrm{~cm} . \mathrm{s}^{-1}$ against for $3.310^{-11} \mathrm{~cm} . \mathrm{s}^{-1}$ [41] [40] for PBSA and PHBV, respectively. It is worth noting that all the polymers used in these studies were of the same commercial grade as those of the present study. Based on these data, one 
can infer that water molecules would diffuse more rapidly in the PBSA film than in the PHBV one. The evolution of the number-average molecular weight of polymer chains reflecting a degradation phenomenon mainly induced by liquid water used for hydrolysis reaction, index scission was found to be higher in the PBSA film than in the PHBV one.

\section{Figure 9}

The next step in the analysis of the degradation-induced changes in the bulk of the polymer films was the investigation of their thermal properties. The results deduced from the DSC measurements of PHBV and PBSA films as a function of degradation time in compost are reported in Figure 10 and 11 . The crystallinity ratio of initial films appeared slightly different with $X c$ value for PBSA being lower $(46.7 \pm 1 \%)$ than for PHBV $(53.6 \pm 0.7 \%)$. Over the first 40 days of incubation in compost the evolution of crystallinity indicated significant differences between both polymers. An increase of crystallinity of $23 \%$ was recorded for PBSA films whereas only a slight increase of $2.4 \%$ was observed for PHBV. These results supported the fact that amorphous phase would be preferentially degraded during composting for PBSA films thus increasing the crystallinity ratio, whereas for PHBV both amorphous and crystalline phase would be equally degraded. It could also be hypothesised that crystals of PHBV would be more easily degraded than the crystals of PBSA due to significant differences in their surface structure, morphology and chain mobility [42].

Iggui et al. [20] also reported a significant reduction of Mw during degradation of PHBV in compost whereas no change in crystallinity was observed. However, no abiotic hydrolysis control was performed to evaluate the respective contribution of hydrolytic and enzymatic degradation. Rutkowska et al. [17] reported that PHBV films degraded via enzymatic process with a slight influence of the hydrolytic process. The weak decrease of $\mathrm{Mw}$ ( $8 \%$ in two weeks) obtained by these authors suggested that the bulk of the material could also be affected during the biodegradation process. But, the hydrolytic process could have been slower due to different experimental composting conditions as uncontrolled parameters and a lower average temperature $\left(21^{\circ} \mathrm{C}\right.$ against $58^{\circ} \mathrm{C}$ in the present study). In contrast, Luo et al. [16] and Weng et al. [18] observed neither change in the $\mathrm{Mw}$, nor changes in crystallinity during degradation in compost. Regarding the degradation-induced changes in compost Puchalski at al. [21] also observed an increase of the crystallinity of PBSA with a concomitant decrease of the molecular weight. These authors concluded that the increase in cristallinity was a result of both the hydrolysis and enzymatic degradation with a degradation of amorphous parts being first followed by crystalline ones.

Besides the assumption based on a differential accessibility of the crystalline phase of both polymers by degrading enzymes that will be developed below, recrystallization phenomenon could also be evoked to account for the increased crystallinity rates during the biodegradation process. As evoked above, water-induced hydrolysis of polymer chains occurred in the bulk of both polymers in the first stage of incubation. Such chain cleavages 
were expected to provide an extra mobility to the oligomers and shortened polymer chains, especially those that are entrapped in the bulk of the film, leading them to crystallise and/or the remaining crystalline phase to reorganise, giving rise to an increase in crystallinity [43].

This assumption was supported by the DSC thermograms of PHBV and PBSA films (Fig. 11), which showed two melting peaks evolving conversely over time, the enthalpy of the first melting peak decreasing while the second one increases. Based on these results one can infer that a reorganization of the crystalline phase of the two polymers occurred during incubation in compost medium. It is worth noting that the rather high temperature $\left(58^{\circ} \mathrm{C}\right)$ set in the compost medium combined with a relative humidity close to $100 \%$ were conditions prone to promote the reorganisation of the crystalline phase. Iggui et al. [44] also reported the formation of a double melting peak and a decrease of the melting temperature of PHBV films subjected to photo-oxidation. These changes were attributed to the formation of new crystal populations with different morphologies and dimensions together with a reduction in molecular weight of PHBV polymer chains. During the degradation process, it could be supposed that the structure of PHBV evolved toward a lower ordered structure of crystal and shorter polymer chains as supported by the decrease of the melting temperatures (Fig. 11). On the contrary, the increase in melting temperature observed for PBSA over the degradation process reflected a lower flexibility of polymer chains and a higher level of structuration of the crystals as reflected by the increased degree of crystallinity. These structural changes could also explain the ability of PBSA to fragment during degradation in compost.

\section{GENERAL DISCUSSION}

As expected PBSA and PHBV films were rapidly biodegraded (60 to 80 days) in lab-scale composting conditions. But, PHBV degraded faster than PBSA in spite of its higher molecular weight (Fig. 8) and degree of crystallinity (Fig. 9) together with quite similar surface hydrophilicity (table 1). This led to suggest that different biodegradation mechanisms would be involved depending on the polymer tested. For this purpose, a two-phase scenario was proposed for each polymer. In both cases, the loss of material appeared mostly driven by enzymatic erosion occurring at the surface of each material with a weaker contribution of hydrolytic chain scission mechanisms induced by water diffusion that mainly affected the bulk of both polymers. It is however worth noting that the mechanism of hydrolytic chain scission was shown to be more pronounced for PBSA than PHBV (Fig. 8) due to significant differences in water permeability, water diffusion and molecular weight.

In the first stage of degradation, PHBV mass loss increased exponentially with the amorphous regions being preferentially degraded as compared to the crystalline ones. SEM observations (Fig. 3) evidenced a very rough surface after primary surface erosion of PHBV film, which revealed the structural organization of its crystals in spherulites. As a result, the surface became porous with an important increase of the specific surface. This was expected to expose more polymer chains at the film surface with as major consequence an increase of 
the enzymatic hydrolysis rate in composting conditions, where the concentration of degrading enzymes is not considered as a limiting factor. As demonstrated by DSC analysis focusing on the bulk (Fig. 10-11), both amorphous and crystalline phase of PHBV seemed to degrade at the same rate resulting in weak changes in crystallinity according to time, the lower degradation rate of the crystalline phase being compensated by its higher area revealed by the enzymatic etching of the film surface. The increase of carbonyl index during this first period also emphasized the significant surface erosion of PHBV (Fig. 7). At the end of this period ( $\approx 20$ days), mass loss and $\mathrm{CO}_{2}$ released measurements led to suppose that the degradation rate reached a steady state with stabilization, and then a slow down at the end of the biodegradation process (Fig. 1-2). This scenario was also in concordance with the evolution of carbonyl index of PHBV, which indicated a decrease during this second phase (Fig. 7).

By contrast, mass loss evolved almost linearly for PBSA during the first phase of degradation (Fig. 1-2). The etched surface evidenced by SEM as a function of the degradation time (Fig. 3) made appear crystals organized in flat layers parallel to the film surface. Such a spatial organization of crystal was not prone to promote surface erosion. The crystalline phase being degraded slower than the amorphous one, the surface erosion of PBSA evolved slowly. The increase of carbonyl index observed during this phase was also consistent with a low erosion rate of the film surface (Fig. 7). As a consequence PBSA film exhibited weak changes in roughness and specific surface during this first phase. However, the propensity of PBSA to become brittle instead of porous gradually led the film to fragment throughout the degradation in compost (Fig. 5-6). At the end of this first phase, surface erosion and fragmentation have sufficiently progressed to increase the exposition of the amorphous regions of PBSA giving them more access to enzymes. This led the degradation kinetic to accelerate in the second phase ( $>20$ days) as evidenced by evolution in mass loss and mineralisation rates (Fig. 1-2). As a consequence, the higher degradation rate of the amorphous phase caused the crystallinity rate of the film to increase sharply. In addition, recrystallisation phenomenon induced by extra mobility of the oligomers and short polymer chains generated by water-induced hydrolysis occurring in the bulk of PBSA also contributed to the increase of crystallinity (Fig. 10-11). The plateau reached by the carbonyl index of PBSA from 20 days emphasized the assumption that the oligomers and short polymer chains produced by ester linkages hydrolysis were rapidly released from the film to be assimilated in the compost medium afterwards as indicated by the mineralization curve (Fig. 2, 7).

Based on these two scenarios, the discrepancy in biodegradation rate between PHBV and PBSA would be essentially attributed to significant differences in crystals morphology and spatial organization of both polymers. Furthermore, it could not be excluded that among the different microbial communities adhering on plastics, some of them possess better hydrolytic capabilities regarding crystalline areas. In that regard, PHBV and PBSA films displayed initially different surface properties, notably roughness and surface topography at $10 \mu \mathrm{m}$ scale (Table 1). Such properties being known to control the adherence of 
microorganisms on a surface, one can infer that the higher roughness of PBSA might hinder the colonization of microorganisms at its surface [31]. This hypothesis was supported by SEM images (Fig. 4) revealing footprints of microbial filaments on the surface of PHBV films whereas these filaments were absent or scarcely observed on PBSA films. This led to suggest that colonization of PHBV by fungi could thus have been more important than for PBSA. This assumption was also supported by Mercier et al. [7], who reported that a higher microbial colonization was observed for mcl-PHA than for PBS. Lastly, as shown by Song et al. [32] material stiffness could also promote the colonization of the surface by microorganisms. The stiffness value of PHBV being greater than these of PBSA with a young modulus of $4200 \mathrm{MPa}$ versus $290 \mathrm{MPa}$, respectively according to the furnisher specifications, this could play in favour of a better propensity of PHBV to be colonized by microorganisms. The importance and better degradation capability of mycelia microorganisms involved in the biodegradation of plastics has already been pointed out [45] [46]. Though, mechanisms of microbial adhesion in relation to polymer surface properties remain still poorly understood and would need further investigations.

\section{Conclusions}

This study was performed to compare biodegradation mechanisms of two well-known polymers and identify the most relevant indicators to follow biodegradation in a given environment. On the strength of the results obtained and the two scenarios proposed, the discrepancy in biodegradation rate between PHBV and PBSA would be essentially attributed to significant differences in crystals morphology and spatial organization of both polymers.

Regarding the relevance of the different indicators studied, mass loss stood out as the most relevant and accurate indicator to assess the disappearance of material. But, it is unavoidable to associate mass loss measurements with mineralization kinetics to attest the complete conversion of the polymer organic carbon into $\mathrm{CO}_{2}$. Besides, SEM and AFM can be seen as powerful tools to evidence surface erosion and morphological changes at different scales. At last, changes in polymer thermal properties were shown to reflect not only surface and bulk degradation, but also recrystallization phenomenon. For that regard, they appear as inescapable tools for better understanding the underlying mechanisms involved in polymer biodegradation. By contrast, the relevance of index carbonyl as indicator of the biodegradation progress appeared less obvious since it can be interpreted differently depending on the capacity of the cleaved polymer chains to be released or not from the film. The same remark can be deduced from molecular weight measurements that mainly reflect water-induced hydrolysis occurring in the material bulk. So, one can infer that none of these two latter indicators is able to assess biodegradation or predict it.

\section{Acknowledgments}

The authors gratefully acknowledge Vincent Darcos (SynBio3, IBMM, Montpellier) for the support provided in GPC analysis and Didier Cot (IEM, Montpellier) for SEM observations. 
[1] Geyer, R., J.R. Jambeck, and K.L. Law. Production, use, and fate of all plastics ever made. Science Advances. 2017. 3(7): e1700782.

[2] Steinbüchel, A. and T. Lütke-Eversloh. Metabolic engineering and pathway construction for biotechnological production of relevant polyhydroxyalkanoates in microorganisms. Biochemical Engineering Journal. 2003. 16(2): 81-96.

[3] Corre, Y.-M., S. Bruzaud, J.-L. Audic, and Y. Grohens. Morphology and functional properties of commercial polyhydroxyalkanoates: A comprehensive and comparative study. Polymer Testing. 2012. 31(2): 226-235.

[4] Jost, V. Packaging related properties of commercially available biopolymers - An overview of the status quo. eXPRESS Polymer Letters. 2018. 12(5): 429-435.

[5] Xu, J. and B.H. Guo. Poly(butylene succinate) and its copolymers: research, development and industrialization. Biotechnol J. 2010. 5(11): 1149-63.

[6] Hoshino, A., H. Sawada, M. Yokota, M. Tsuji, K. Fukuda, and M. Kimura. Influence of weather conditions and soil properties on degradation of biodegradable plastics in soil AU - Hoshino, Akira. Soil Science and Plant Nutrition. 2001. 47(1): 35-43.

[7] Mercier, A., K. Gravouil, W. Aucher, S. Brosset-Vincent, L. Kadri, J. Colas, D. Bouchon, and T. Ferreira. Fate of Eight Different Polymers under Uncontrolled Composting Conditions: Relationships Between Deterioration, Biofilm Formation, and the Material Surface Properties. Environmental Science \& Technology. 2017. 51(4): 1988-1997.

[8] Yang, H.-S., J.-S. Yoon, and M.-N. Kim. Dependence of biodegradability of plastics in compost on the shape of specimens. Polymer Degradation and Stability. 2005. 87(1): 131-135.

[9] Avella, M., E. Martuscelli, and M. Raimo. Review Properties of blends and composites based on poly(3-hydroxy)butyrate (PHB) and poly(3-hydroxybutyrate-hydroxyvalerate) (PHBV) copolymers. Journal of Materials Science. 2000. 35(3): 523-545.

[10] Mergaert, J., C. Anderson, A. Wouters, J. Swings, and K. Kersters. Biodegradation of polyhydroxyalkanoates. FEMS Microbiology Letters. 1992. 103(2): 317-321.

[11] Tserki, V., P. Matzinos, E. Pavlidou, D. Vachliotis, and C. Panayiotou. Biodegradable aliphatic polyesters. Part I. Properties and biodegradation of poly(butylene succinate-co-butylene adipate). Polymer Degradation and Stability. 2006. 91(2): 367-376.

[12] Bugnicourt, E., P. Cinelli, V. Alvarez, and A. Lazzeri. Polyhydroxyalkanoate (PHA): Review of synthesis, characteristics, processing and potential applications in packaging. eXPRESS Polymer Letters. 2014. 8(11): 791-808.

[13] Tokiwa, Y., B.P. Calabia, C.U. Ugwu, and S. Aiba. Biodegradability of plastics. International Journal of Molecular Sciences. 2009. 10(9): 3722-3742.

[14] Lucas, N., C. Bienaime, C. Belloy, M. Queneudec, F. Silvestre, and J.-E. Nava-Saucedo. Polymer biodegradation: Mechanisms and estimation techniques - A review. Chemosphere. 2008. 73(4): 429-442.

[15] Eldsäter, C., S. Karlsson, and A.-C. Albertsson. Effect of abiotic factors on the degradation of poly(3-hydroxybutyrate-co-3-hydroxyvalerate) in simulated and natural composting environments. Polymer Degradation and Stability. 1999. 64(2): 177-183.

[16] Luo, S. and A.N. Netravali. A study of physical and mechanical properties of poly(hydroxybutyrate-co-hydroxyvalerate) during composting. Polymer Degradation and Stability. 2003. 80(1): 59-66.

[17] Rutkowska, M., K. Krasowska, A. Heimowska, G. Adamus, M. Sobota, M. Musioł, H. Janeczek, W. Sikorska, A. Krzan, E. Žagar, and M. Kowalczuk. Environmental Degradation of Blends of Atactic Poly[(R,S)-3-hydroxybutyrate] with Natural PHBV in Baltic Sea Water and Compost with Activated Sludge. Journal of Polymers and the Environment. 2008. 16(3): 183-191.

[18] Weng, Y.-X., Y. Wang, X.-L. Wang, and Y.-Z. Wang. Biodegradation behavior of PHBV films in a pilot-scale composting condition. Polymer Testing. 2010. 29(5): 579-587. 
[19] Weng, Y.-X., X.-L. Wang, and Y.-Z. Wang. Biodegradation behavior of PHAs with different chemical structures under controlled composting conditions. Polymer Testing. 2011. 30(4): 372-380.

[20] Iggui, K., N. Le Moigne, M. Kaci, S. Cambe, J.-R. Degorce-Dumas, and A. Bergeret. A biodegradation study of poly(3-hydroxybutyrate-co-3-hydroxyvalerate)/organoclay nanocomposites in various environmental conditions. Polymer Degradation and Stability. 2015. 119: 77-86.

[21] Puchalski, M., G. Szparaga, T. Biela, A. Gutowska, S. Sztajnowski, and I. Krucińska. Molecular and Supramolecular Changes in Polybutylene Succinate (PBS) and Polybutylene Succinate Adipate (PBSA) Copolymer during Degradation in Various Environmental Conditions. 2018. 10(3): 251.

[22] Deroine, M., G. Cesar, A. Le Duigou, P. Davies, and S. Bruzaud. Natural Degradation and Biodegradation of Poly(3-Hydroxybutyrate-co-3-Hydroxyvalerate) in Liquid and Solid Marine Environments. Journal of Polymers and the Environment. 2015. 23(4): 493-505.

[23] Owens, D.K. and R.C. Wendt. Estimation of the surface free energy of polymers. Journal of Applied Polymer Science. 1969. 13(8): 1741-1747.

[24] Van Oss, C.J., R.J. Good, and M.K. Chaudhury. Additive and nonadditive surface tension components and the interpretation of contact angles. Langmuir. 1988. 4(4): 884-891.

[25] Siracusa, V., N. Lotti, A. Munari, and M. Dalla Rosa. Poly(butylene succinate) and poly(butylene succinate-co-adipate) for food packaging applications: Gas barrier properties after stressed treatments. Polymer Degradation and Stability. 2015. 119: 35-45.

[26] Kann, Y., M. Shurgalin, and R.K. Krishnaswamy. FTIR spectroscopy for analysis of crystallinity of poly(3-hydroxybutyrate-co-4 -hydroxybutyrate) polymers and its utilization in evaluation of aging, orientation and composition. Polymer Testing. 2014. 40: 218-224.

[27] Sedlacek, P., E. Slaninova, V. Enev, M. Koller, J. Nebesárová, I. Marova, K. Hrubanova, V. Krzyzanek, O. Samek, and S. Obruca. What keeps polyhydroxyalkanoates in bacterial cells amorphous? A derivation from stress exposure experiments. Applied Microbiology and Biotechnology. 2019. 103(4): 1905-1917.

[28] Nguyen, T.Q. Kinetics of mechanochemical degradation by gel permeation chromatography. Polymer Degradation and Stability. 1994. 46(1): 99-111.

[29] Charlon, S., N. Follain, C. Chappey, E. Dargent, J. Soulestin, M. Sclavons, and S. Marais. Improvement of barrier properties of bio-based polyester nanocomposite membranes by water-assisted extrusion. Journal of Membrane Science. 2015. 496: 185-198.

[30] Rosa, D.S., N.T. Lotto, D.R. Lopes, and C.G.F. Guedes. The use of roughness for evaluating the biodegradation of poly- $\beta$-(hydroxybutyrate) and poly- $\beta$-(hydroxybutyrate-co- $\beta$-valerate). Polymer Testing. 2004. 23(1): 3-8.

[31] Riedewald, F. Bacterial Adhesion to Surfaces: The Influence of Surface Roughness. PDA Journal of Pharmaceutical Science and Technology. 2006. 60(3): 164-171.

[32] Song, F., H. Koo, and D. Ren. Effects of Material Properties on Bacterial Adhesion and Biofilm Formation. J Dent Res. 2015. 94(8): 1027-34.

[33] Nishida, H. and Y. Tokiwa. Effects of higher-order structure of poly(3-hydroxybutyrate) on its biodegradation. II. Effects of crystal structure on microbial degradation. Journal of environmental polymer degradation. 1993. 1(1): 65-80.

[34] Kijchavengkul, T., R. Auras, M. Rubino, S. Selke, M. Ngouajio, and R.T. Fernandez. Biodegradation and hydrolysis rate of aliphatic aromatic polyester. Polymer Degradation and Stability. 2010. 95(12): 2641-2647.

[35] Deroiné, M., A. Le Duigou, Y.-M. Corre, P.-Y. Le Gac, P. Davies, G. César, and S. Bruzaud. Accelerated ageing and lifetime prediction of poly(3-hydroxybutyrate-co-3-hydroxyvalerate) in distilled water. Polymer Testing. 2014. 39: 70-78.

[36] Wei, L. and A.G. McDonald. Accelerated weathering studies on the bioplastic, poly(3hydroxybutyrate-co-3-hydroxyvalerate). Polymer Degradation and Stability. 2016. 126: 93100. 
[37] Han, X. and J. Pan. Polymer chain scission, oligomer production and diffusion: A two-scale model for degradation of bioresorbable polyesters. Acta Biomaterialia. 2011. 7(2): 538-547.

[38] Gleadall, A. and J. Pan. Computer simulation of polymer chain scission in biodegradable polymers. Journal of biotechnology and biomaterials. 2013. 3(1):154-159

[39] Charlon, S., Follain, N. , Soulestin, Jérémie ; Sclavons, Michel and S. Marais. Water Transport Properties of Poly(butylene succinate) and Poly[(butylene succinate)-co-(butylene adipate)] Nanocomposite Films: Influence of the Water-Assisted Extrusion Process. The Journal of Physical Chemistry Part C: Nanomaterials and Interfaces. 2017.121:918-930

[40] Crétois, R., Follain, N., Dargent, E., Soulestin, J., Bourbigot S., Marais, S., and L. Lebrun. Microstructure and barrier properties of PHBV/organoclays bionanocomposites. Journal of Membrane Science. 2014.467:56-66

[41] Messin, T., Follain, N., Guinault, A., Sollogoub, C., Gaucher, V., Delpouve, N., and S. Marais. Structure and barrier properties of multinanolayered biodegradable PLA/PBSA films: confinement effect via forced assembly coextrusion. ACS Applied Materials and Interfaces. 2017.9(34):29101-29112.

[42] Gan, Z., K. Kuwabara, H. Abe, T. Iwata, and Y. Doi. The role of polymorphic crystal structure and morphology in enzymatic degradation of melt-crystallized poly(butylene adipate) films. Polymer Degradation and Stability. 2005. 87(1): 191-199.

[43] Han, X. and J. Pan. A model for simultaneous crystallisation and biodegradation of biodegradable polymers. Biomaterials. 2009. 30(3): 423-430.

[44] Iggui, K., M. Kaci, N.L. Moigne, and A. Bergeret. The effects of accelerated photooxidation on molecular weight and thermal and mechanical properties of PHBV/Cloisite 30B bionanocomposites. Journal of Renewable Materials. 2018. 6(3): 288-298.

[45] Tosin, M., M. Weber, M. Siotto, C. Lott, and F. Degli Innocenti. Laboratory Test Methods to Determine the Degradation of Plastics in Marine Environmental Conditions. Frontiers in Microbiology. 2012. 3: 225.

[46] Sang, B.-I., K. Hori, Y. Tanji, and H. Unno. Fungal contribution to in situ biodegradation of poly(3hydroxybutyrate-co-3-hydroxyvalerate) film in soil. Applied Microbiology and Biotechnology. 2002. 58(2): 241-7.

\section{LEGEND OF FIGURES}

Figure 1: Evolution of the weight loss (\%) of PBSA and PHBV films during incubation in laboratoryscale compost conditions.

Figure 2: Mineralisation (\%) of PHBV and PBSA films evaluated by monitoring $\mathrm{CO}_{2}$ released during incubation in laboratory-scale compost conditions.

Figure 3: SEM observations of the surface morphology of PHBV and PBSA films collected from the compost medium at day 6, 10 and 20.

Figure 4: SEM images of PHBV and PBSA films surface evidencing footprints of microbial filaments. Figure 5: Pictures of PHBV and PBSA films collected from the compost medium after 20 of incubation Figure 6: SEM observation of PHBV and PBSA films collected from the compost medium at day 41.

Figure 7: Evolution of carbonyl index of PHBV and PBSA films during incubation in compost medium. Carbonyl indexes were calculated using reference peaks at $1379 \mathrm{~cm}^{-1}(-\mathrm{CH} 3$ symmetric wagging) for $\mathrm{PHBV}$ and at $1473 \mathrm{~cm}^{-1}$ for PBSA (-CH- symmetric deformation) 
757 Figure 8: Evolution of the number-average molecular weight (Mn) of PHBV and PBSA films during 758 incubation in laboratory-scale compost conditions

759 Figure 9: Evolution of the scission index (SI) of PHBV and PBSA films during incubation in laboratory760 scale compost conditions

761 Figure 10: Evolution of the crystallinity rate ${ }_{c}(\%)$ of PHBV and PBSA films during incubation in 762 laboratory-scale compost conditions

763 Figure 11: DSC melting peaks of PHBV (a) and PBSA (b) films as a function of degradation time in 764 laboratory-scale compost conditions

765 Figure 1

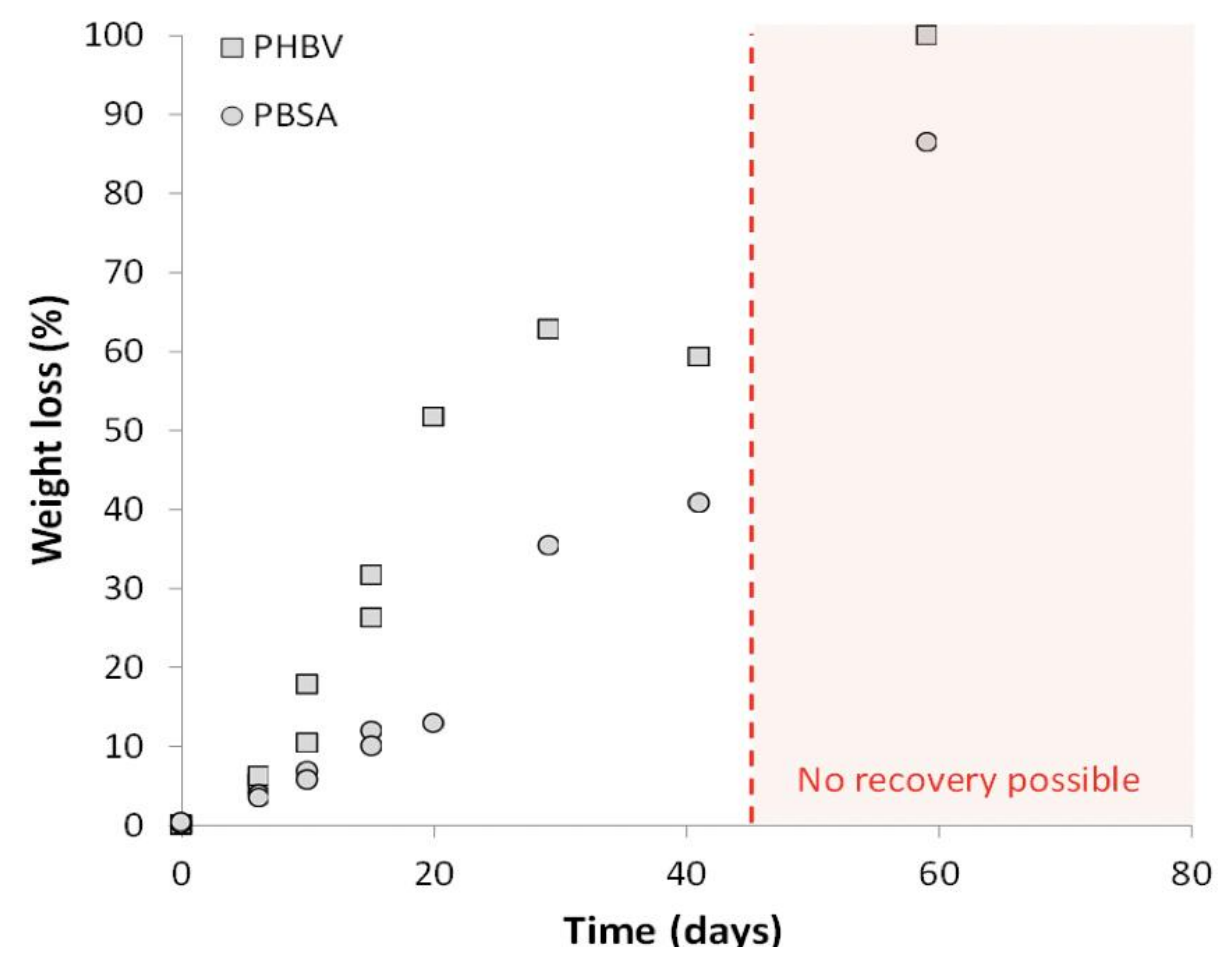

767 Figure 2 


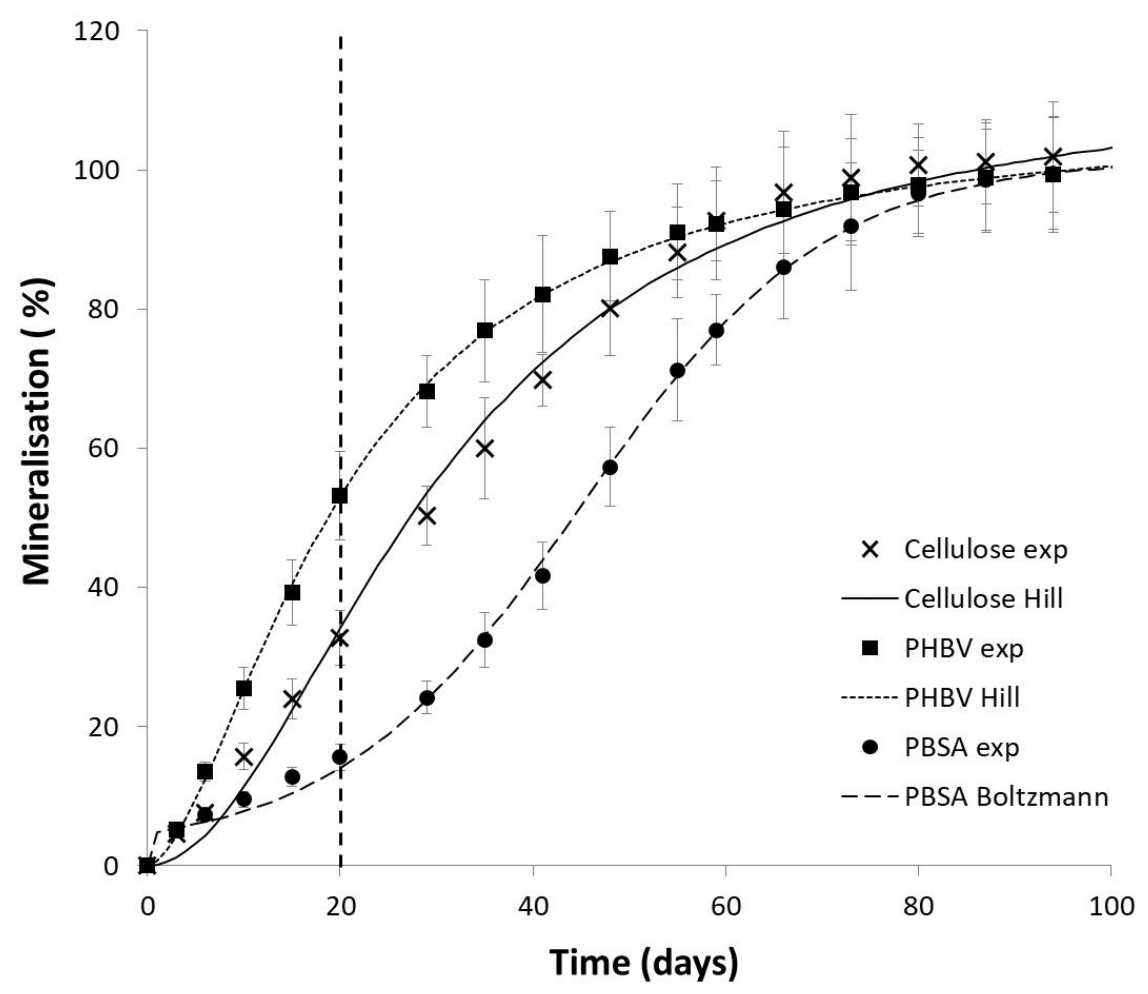

769 Figure 3 


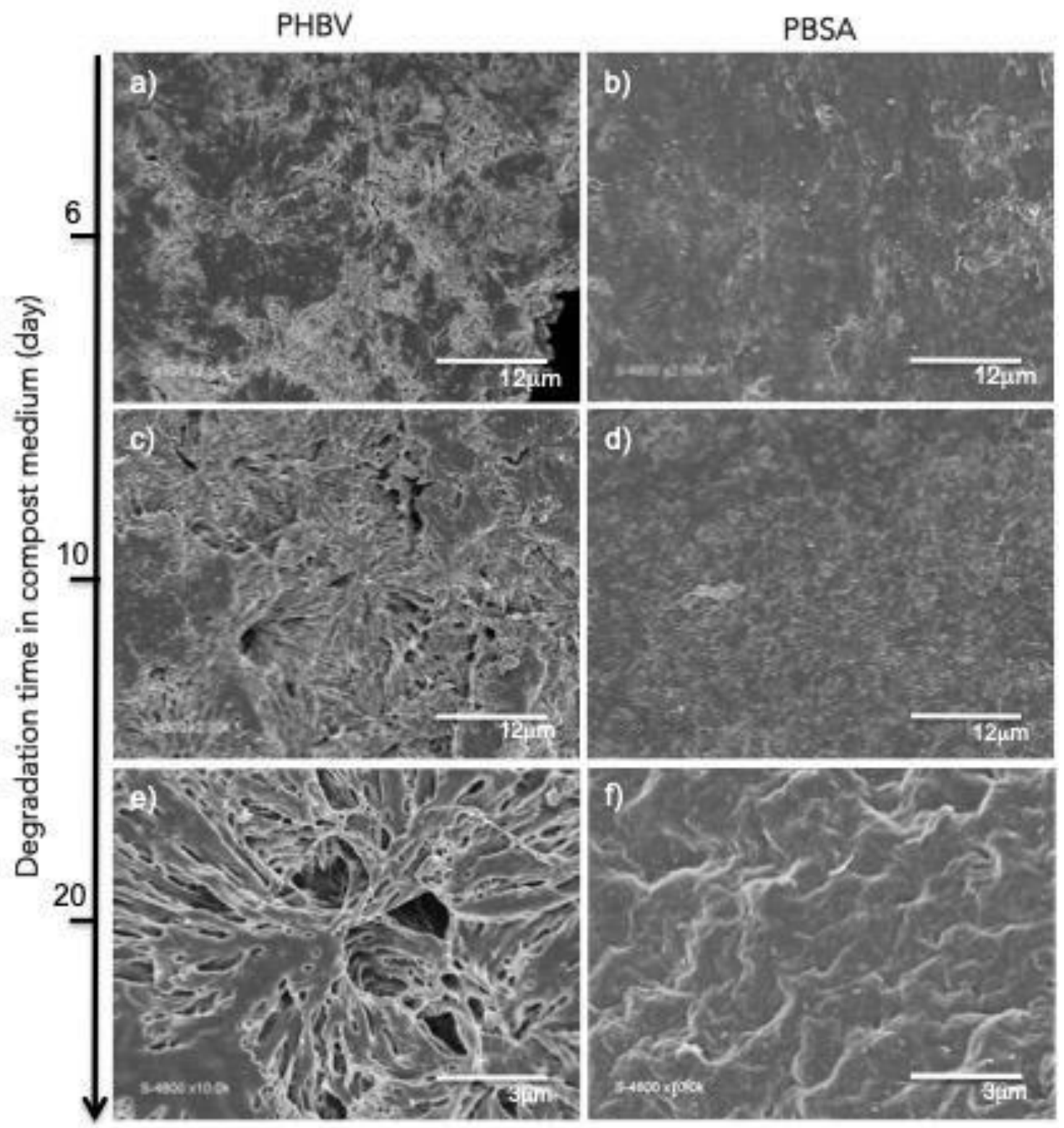

770

771 Figure 4 


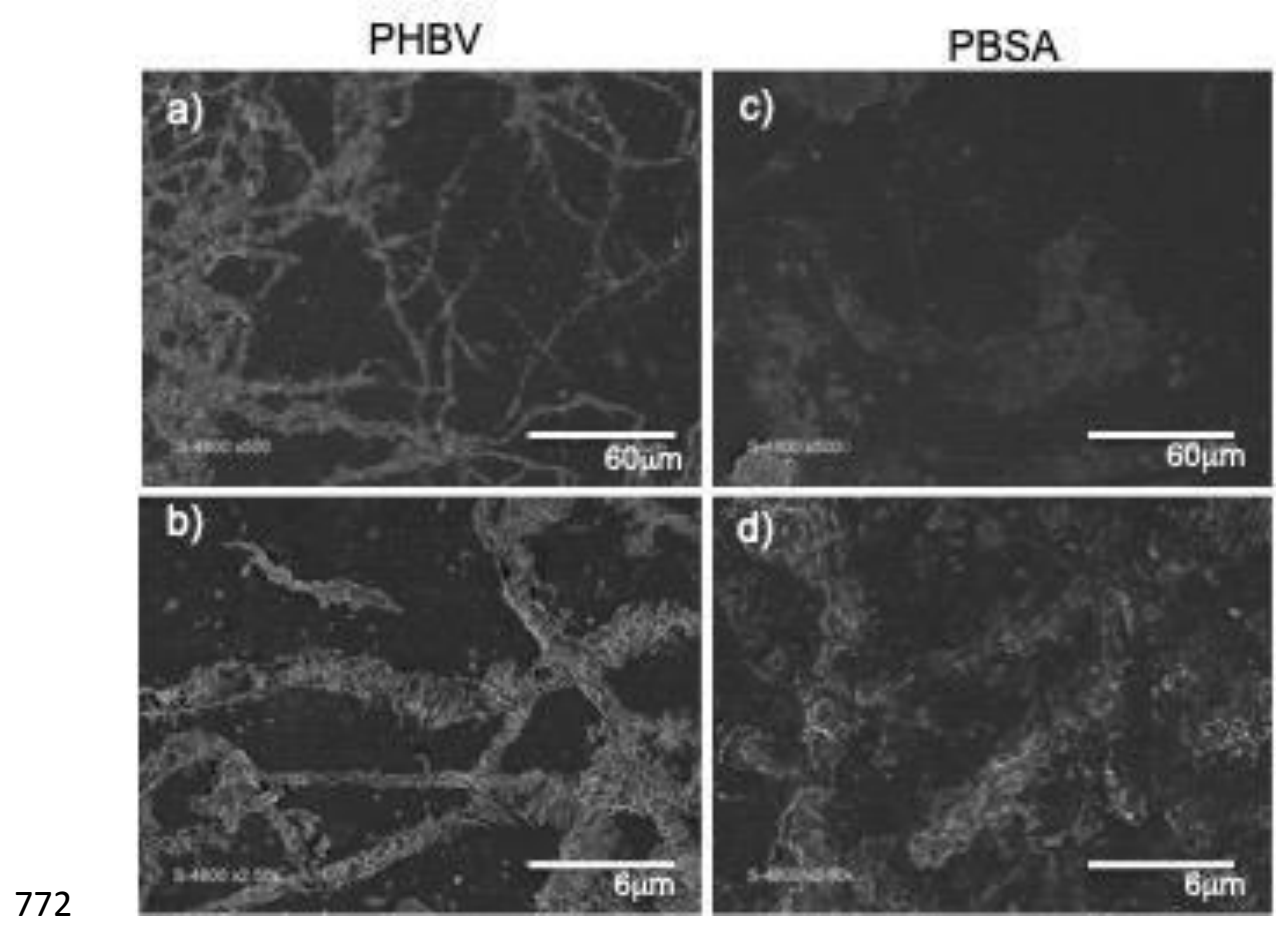

$773 \quad$ Figure 5

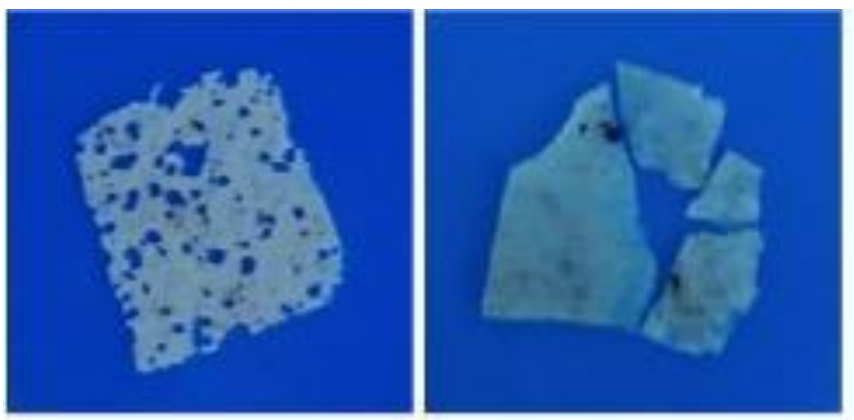

774

$775 \quad$ Figure 6 


\section{PHBV}

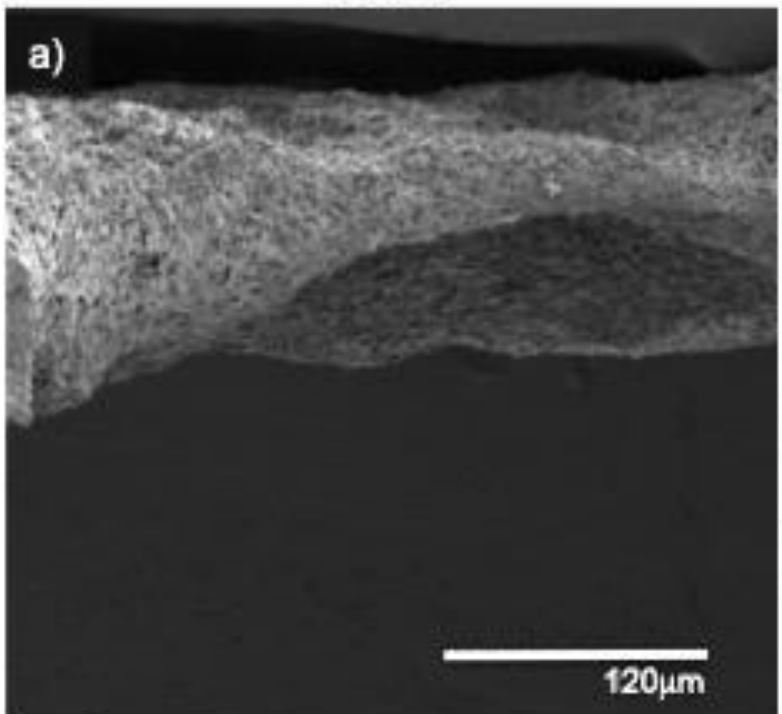

776

777

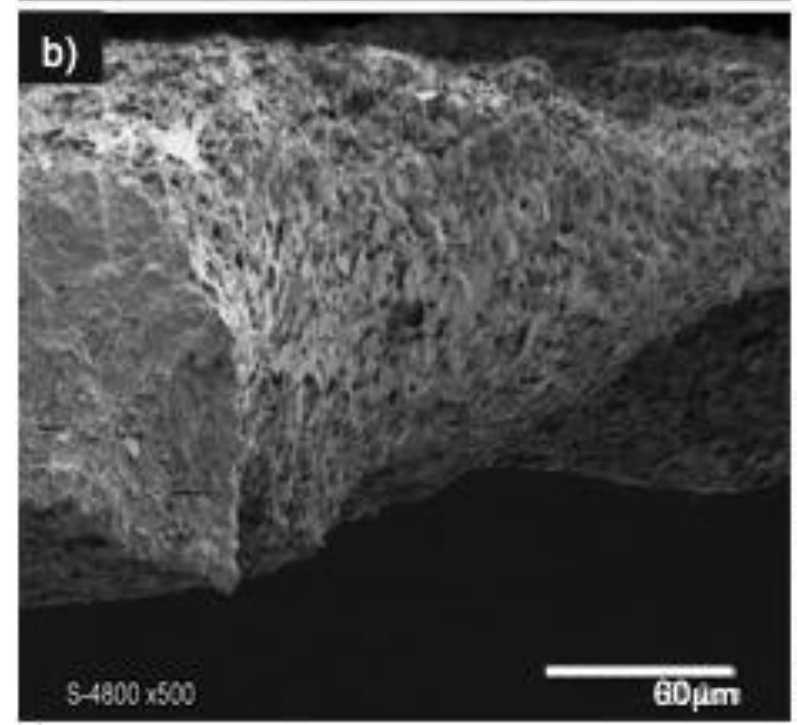

\section{PBSA}

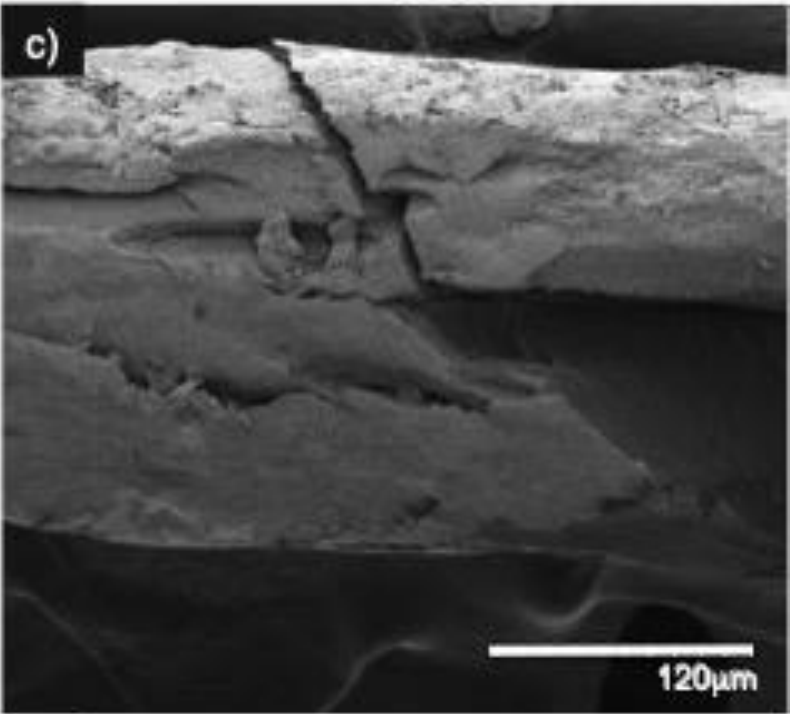

d)

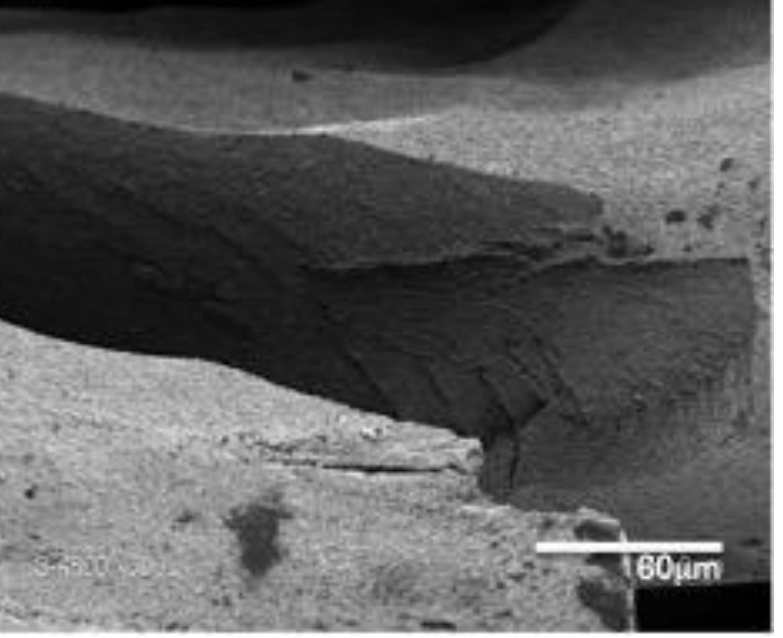

778 Figure 7 
a)

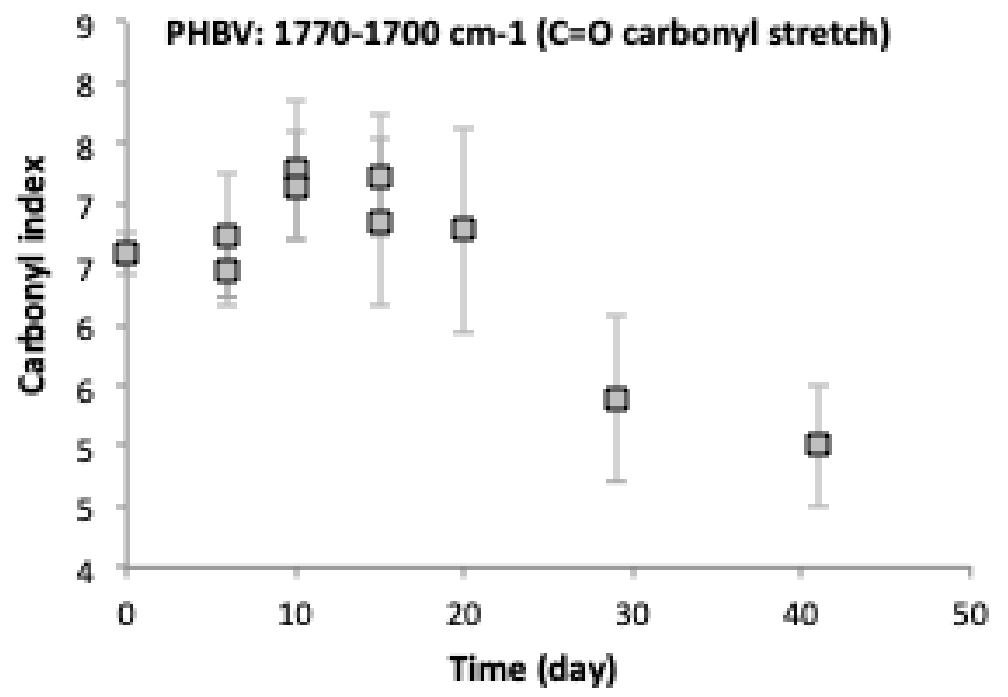

b)

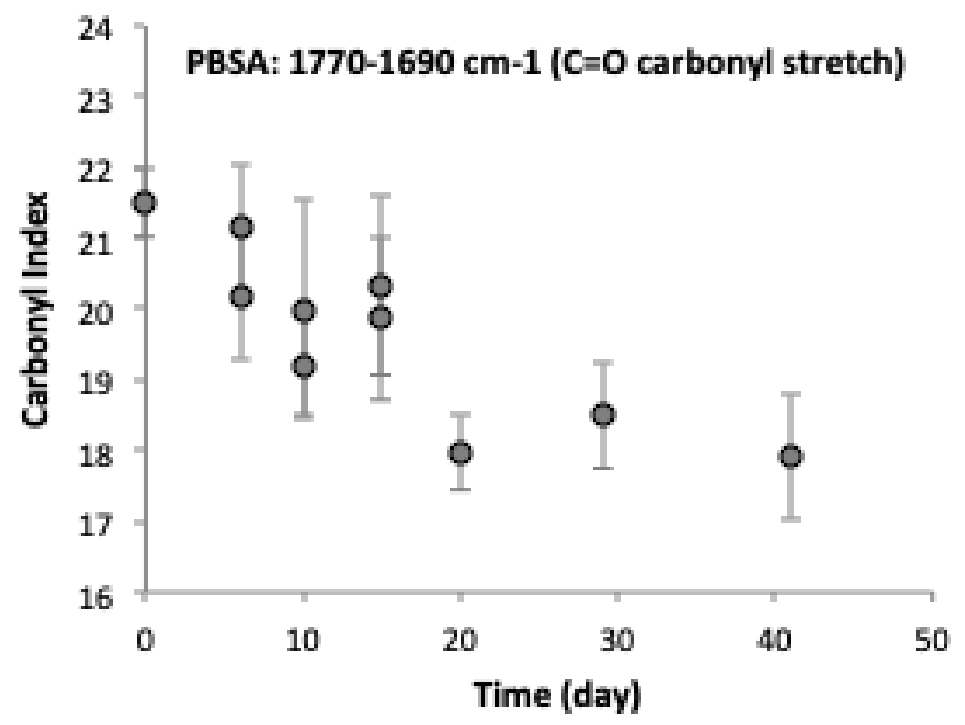

779

Figure 8 


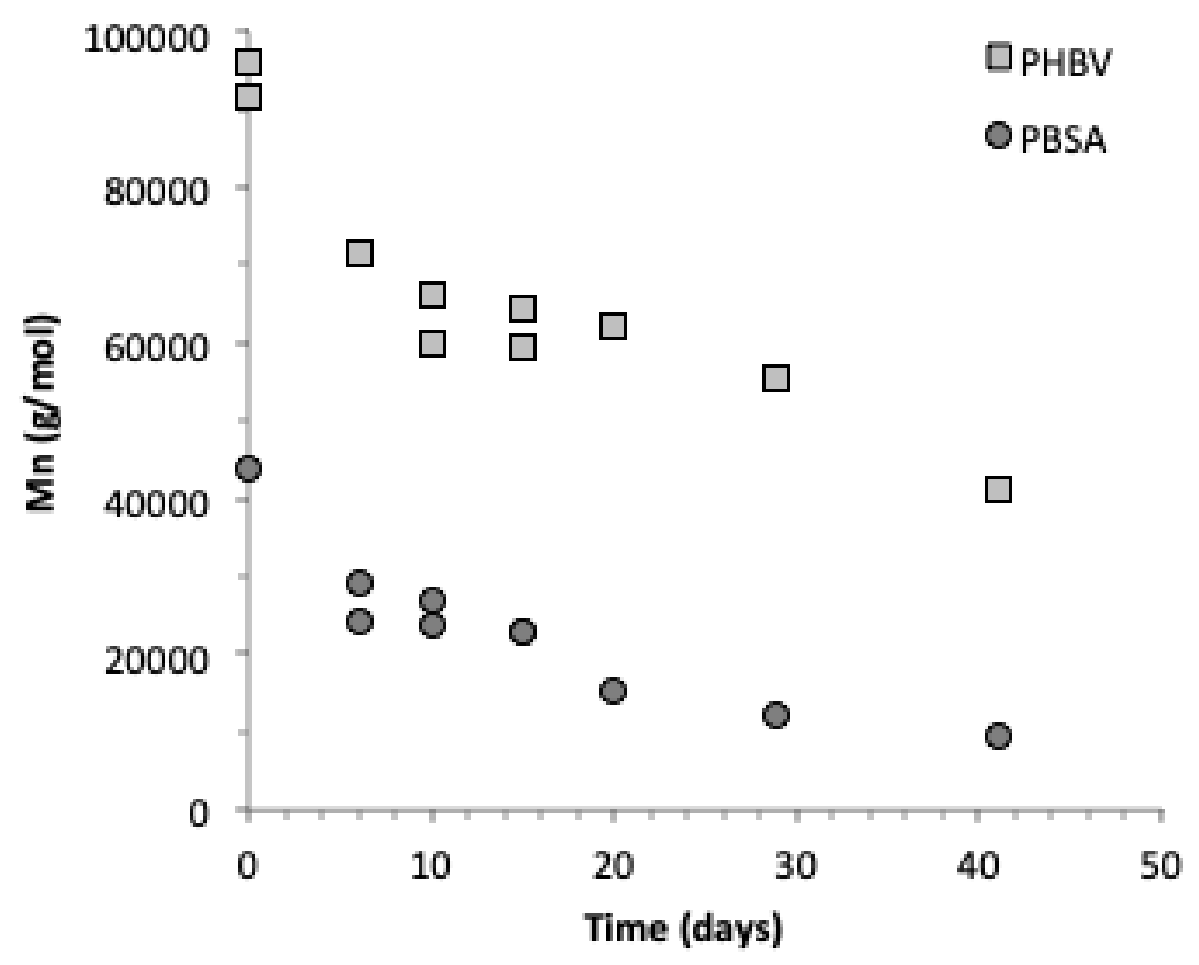

781

782 Figure 9

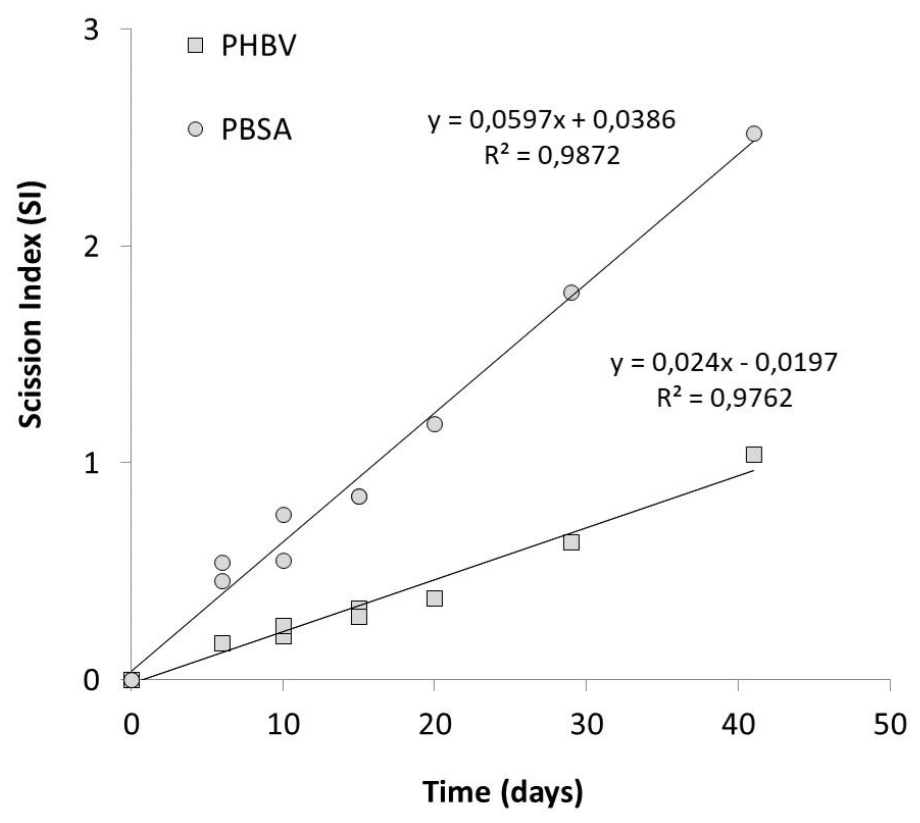

783

784 Figure 10 


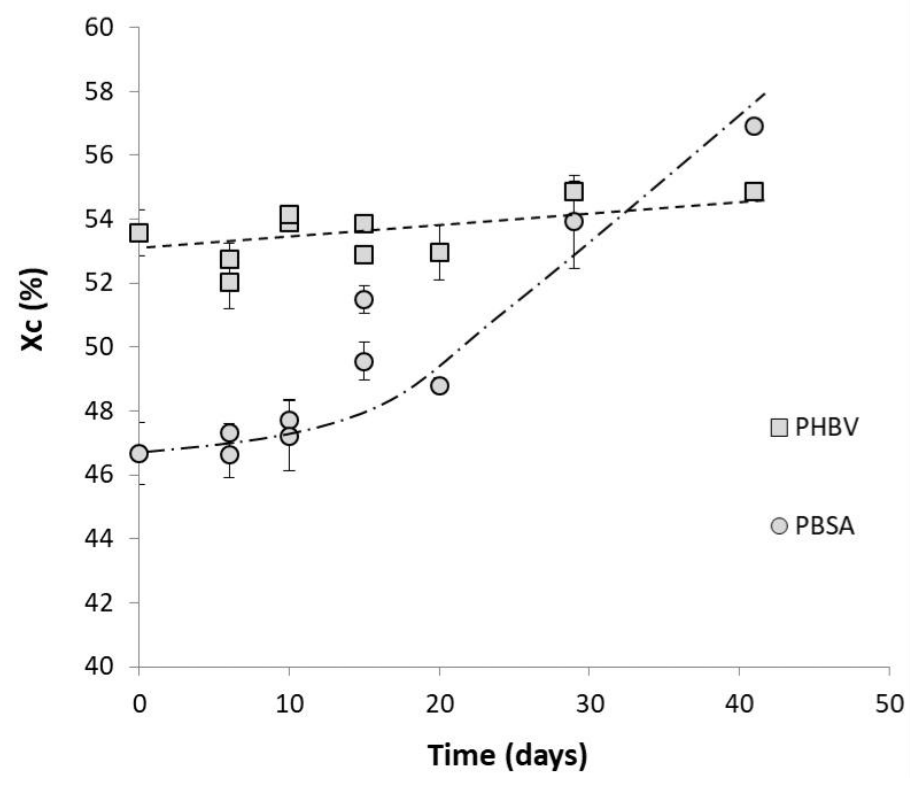

786 Figure 11

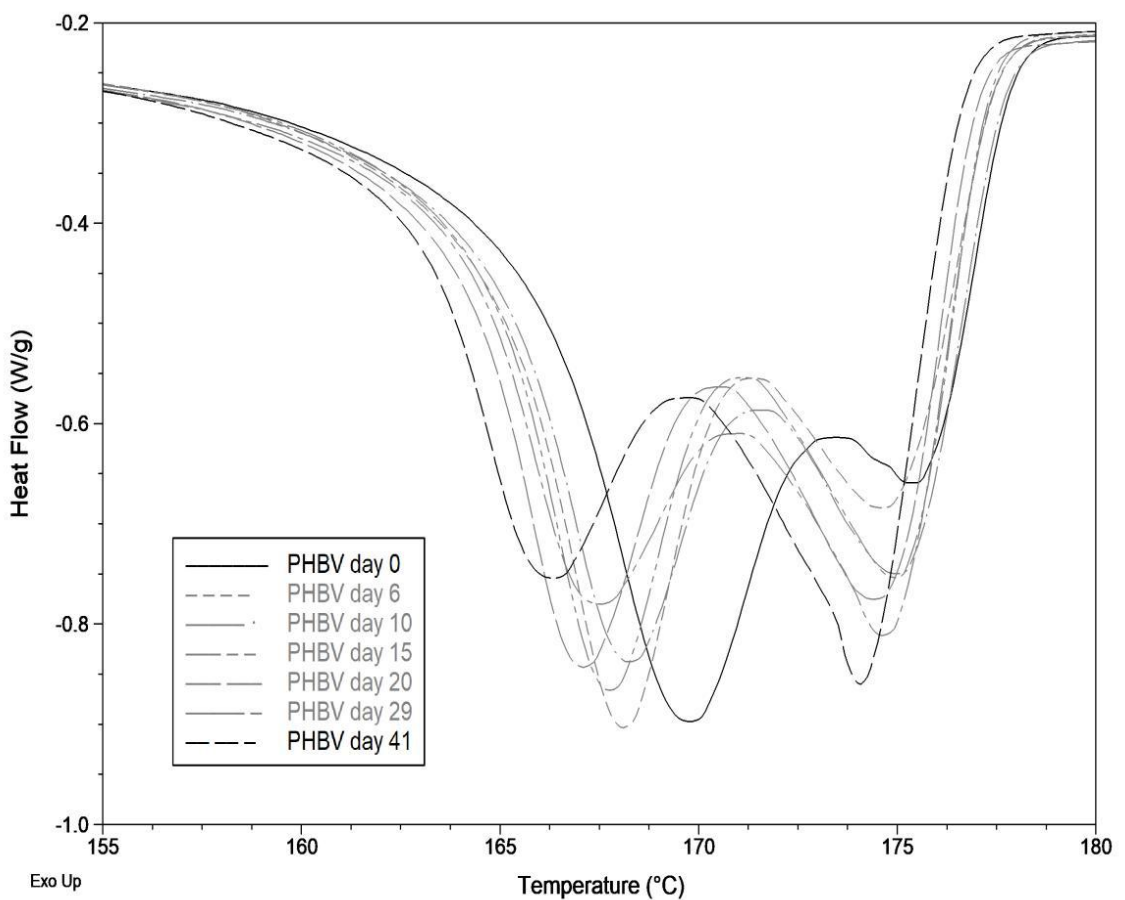

787

788

a) PHBV 


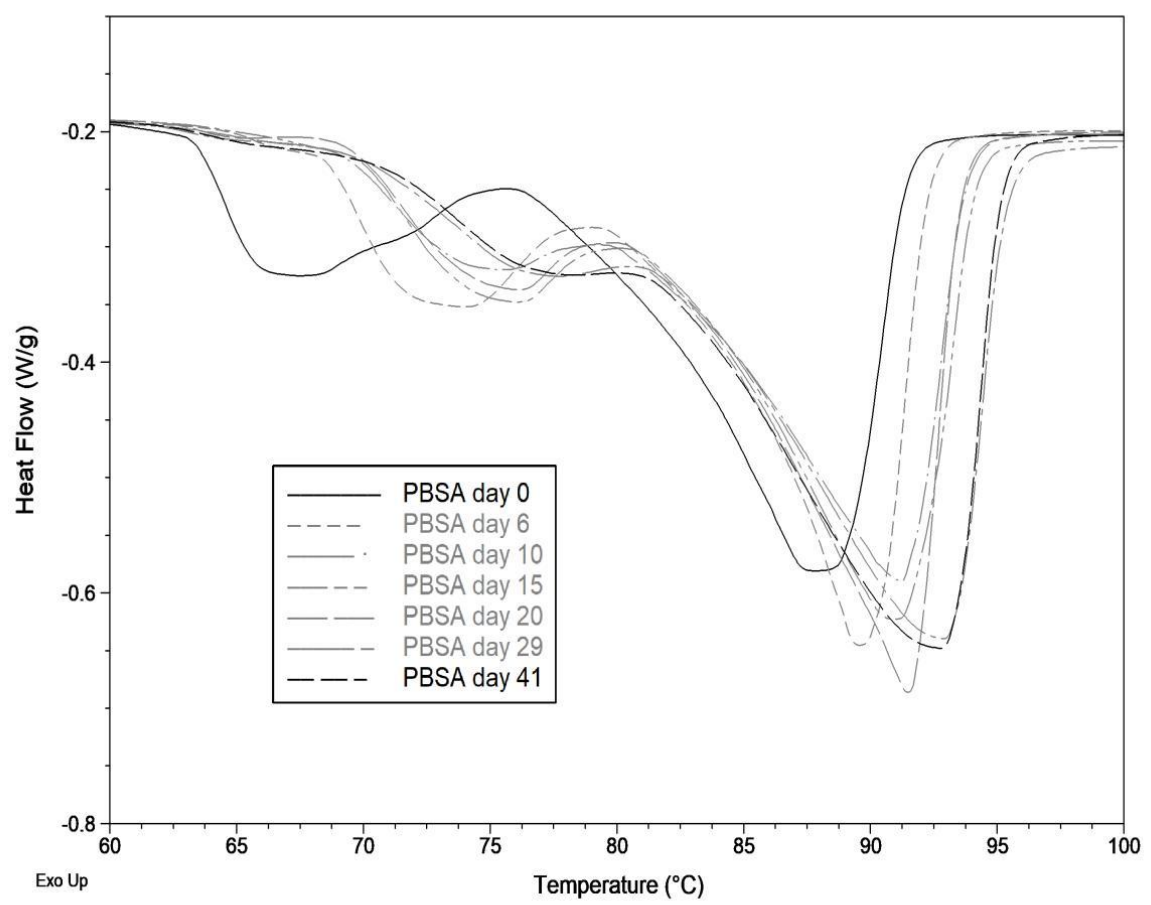

